\title{
The effects of glycaemic variability on intimal hyperplasia and plaque stability after stenting via autophagy-mediated G3BP1/ NLRP3 inflammasome
}

\author{
Jinggang Xia ${ }^{1}$, Jia Zhang ${ }^{2}$, Jing Chang ${ }^{3}$, Yi Tian ${ }^{4}$, Jubo Li ${ }^{5}$, Baojie Zhang ${ }^{5}$, Xiangjun Zeng ${ }^{2}$, Chunlin Yin ${ }^{1}$ \\ ${ }^{1}$ Department of Cardiology, Xuanwu Hospital, Capital Medical University, National Clinical Research Center for Geriatric Diseases, Beijing, \\ China; ${ }^{2}$ Department of Physiology and Pathophysiology, Capital Medical University, Beijing, China; ${ }^{3}$ Department of Pathology, Beijing Youan \\ Hospital, Capital Medical University, Beijing, China; ${ }^{4}$ Beijing Anzhen Hospital, Capital Medical University, Beijing, China; ${ }^{5}$ Department of Animal \\ Experimental Center, Fuwai Hospital, National Center for Cardiovascular Disease, China Academy of Medical Sciences, Beijing, China \\ Contributions: (I) Conception and design: J Xia, X Zeng, C Yin; (II) Administrative support: Y Tian, J Li, B Zhang; (III) Provision of study materials: \\ J Zhang, J Chang; (IV) Collection and assembly of data: J Xia, J Zhang, J Chang; (V) Data analysis and interpretation: All authors; (VI) Manuscript \\ writing: All authors; (VII) Final approval of manuscript: All authors. \\ Correspondence to: Xiangjun Zeng. Department of Physiology and Pathophysiology, Capital Medical University, Beijing China. \\ Email: megan_zeng@163.com; Chunlin Yin. Department of Cardiology, Xuanwu Hospital, Capital Medical University, National Clinical Research \\ Center for Geriatric Diseases, Beijing, China. Email: yinclmaill@163.com.
}

Background: The objective of this study was to investigate the effects of glycaemic variability (GV) on intimal hyperplasia and plaque stability after coronary stenting via autophagy-mediated G3BP1/NLRP3 inflammasome signalling.

Methods: In the clinical study, between July 2017 and December 2017, 95 patients with acute myocardial infarction (AMI) and diabetes mellitus (DM) comorbidity received stent implantation. The patients were followed up for 2 years after discharge. The patients were divided into a low-GV (n=61) and high-GV (n=34) group, and the incidence of recurrent AMI was measured. In the animal study, thirteen pigs were divided into a sham $(\mathrm{n}=3)$, low-GV DM $(\mathrm{n}=5)$ and high-GV DM group $(\mathrm{n}=5)$. Intima samples were analysed by optical coherence tomography 22 weeks after coronary stenting. Becn1, LC3B, p62, G3BP1 and NLRP3 protein levels in the intima were examined by western blot. In vitro experiments with THP-1 cells were also conducted.

Results: In the high-GV group, patients exhibited a higher recurrent AMI, greater neointimal thickness, increased p62 and NLRP3 expression, and decreased Becn1, LC3B and G3BP1 expression compared with the low-GV group $(\mathrm{P}<0.05)$. The effects of high $\mathrm{GV}$ could be abolished by rapamycin but were aggravated by 3-methyladenine.

Conclusions: GV might impact the intimal hyperplasia and plaque stability via autophagy-mediated G3BP1/NLRP3 inflammasome signalling. GV and the autophagy-mediated G3BP1/NLRP3 inflammasome may be promising targets for the treatment of coronary heart disease.

Keywords: Glycaemic variability (GV); diabetes; coronary heart disease (CHD); intima; plaque stability; autophagy; G3BP1; NLRP3 inflammasome

Submitted Jun 19, 2020. Accepted for publication Sep 11, 2020.

doi: 10.21037/atm-20-4818

View this article at: http://dx.doi.org/10.21037/atm-20-4818 


\section{Introduction}

As a major clinical form of coronary heart disease (CHD), acute myocardial infarction (AMI) is critically dangerous to human health. Diabetes is a risk factor for CHD and worsens the prognosis of AMI. In recent years, different blood glucose fluctuations have been shown to lead to different outcomes, even at the same levels of glycated haemoglobin (HbA1c) (1). Glycaemic variability (GV) refers to the fluctuations in blood glucose concentration over time and has attracted much recent attention. The impact of GV on atherosclerosis and the mechanisms by which it affects the development of atherosclerosis remain unclear. Autophagy occurs in the lysosome of eukaryotic cells and is an evolutionarily conserved catabolic programme that deals with protein aggregates and extraneous organelles. Autophagy promotes cellular development and differentiation and maintains homeostasis in response to a variety of pathophysiological stresses (2). The dysfunction of autophagy is reportedly related to atherosclerosis pathogenesis. The role of autophagy in the diabetic heart might be either protective or harmful and is highly controversial $(3,4)$. According to increasingly reliable evidence, GTPase-activating protein ( $\mathrm{SH} 3$ domain)-binding protein 1 (G3BP1) (a critical element and common marker of stress granules) and the NOD-like receptor family pyrin domain-containing 3 (NLRP3) inflammasome are crucial for controlling the physiological processes of stressed cells $(5,6)$. The NLRP3 inflammasome is a multiprotein innate immune complex formed in cytosolic compartments and can detect endogenous danger signals, facilitating the activation of inflammatory protease caspase- 1 and contributing to the progress of CHD (5). Stress granules are conserved cytoplasmic assemblies that facilitate cell survival by modulating a wide range of stress responses (6). Autophagy has been shown to negatively regulate the activation of the NLRP3 inflammasome $(7,8)$. The interplay between autophagy and stress granules has not been studied in detail, and there is little evidence on whether GV plays a role in atherosclerosis via autophagy and G3BP1/NLRP3 inflammasome signalling. To address this knowledge gap, clinical research and animal experiments were performed in the current study. Macrophages have profound effects on atherosclerotic plaque formation and plaque rupture. Thus, we also carried out experiments with human myeloid leukaemia mononuclear (THP-1) cells. This study investigated the impact of $\mathrm{GV}$ on intimal hyperplasia and plaque stability after coronary stenting by exploring the potential interplay signalling between autophagy and the G3BP1/NLRP3 inflammasome. This article is in accordance with the ARRIVE reporting checklist (available at http://dx.doi.org/10.21037/atm-20-4818).

\section{Methods}

\section{Clinical research}

\section{Study population}

This was an observational study to investigate the effects of GV on recurrent acute myocardial infarction (RAMI) in patients with diabetes comorbidity during a 2-year followup using intelligent blood glucose meters. Consecutive diabetic patients with their first AMI who received complete revascularization with percutaneous coronary intervention (PCI) and who were discharged in a stable condition were screened. All procedures were conducted in accordance with the Declaration of Helsinki (as revised in 2013). Ethical approval for the study (ID: 2017-042) was obtained from the Ethics Committee of Xuanwu Hospital of Capital Medical University, and written informed consent was provided by patients before participating in the study.

AMI was defined as the clinical evidence of ischaemic symptoms (>20 min) in combination with ST-segment elevation or the absence of ST elevation and a rise in cardiac-specific troponin I values. Patients without chest tightness and chest pain were determined to be in a stable condition and were discharged by the doctor. The exclusion criteria were as follows: (I) history of myocardial infarction or ECG suggesting previous myocardial infarction; (II) history of coronary artery bypass surgery and those who underwent coronary artery bypass grafting during hospitalization due to coronary disease in three vessels; (III) history of chronic obstructive pulmonary disease with sudden aggravation of respiratory symptoms; (IV) kidney failure identified as serum creatinine greater than $3 \mathrm{mg} / \mathrm{dL}$ or glomerular filtration rate estimation less than $30 \mathrm{~mL} / \mathrm{min} / 1.73 \mathrm{~m}^{2}$ or the need for renal replacement therapy; (V) peptic ulcer disease with or without gastrointestinal bleeding; (VI) history of hyperthyroidism; (VII) stroke within 30 days; (VIII) other severe comorbidities or disorders with an estimated survival time $<2$ years; and (IX) refusal to participate or failure to respond to the invitation after trial initiation.

\section{Study protocols}

Patients who were eligible for enrolment were treated 
with standard medication therapy and PCI during hospitalization. All patients and their caregivers were required to learn how to use an intelligent blood glucose meter application (GLUPAD, SINOMEDISITE, China) by undergoing a training programme. They were instructed how to download, install the application and set up an account on a cell phone and how to measure fingertip blood glucose with the intelligent blood glucose meter. The blood glucose measured by the intelligent meter was automatically uploaded to the cell phones of the doctor and patient. In addition, the use of the application allowed communication between doctors and patients. Patients were instructed to measure their blood glucose at least one day per week at four time points-before breakfast and $2 \mathrm{~h}$ after each of three meals. The primary end point was the incidence of RAMI after 2 years of follow-up.

\section{Grouping}

At the end of the follow-up period, all finger-prick glucose data from each patient were collected, and the mean and standard deviation (SD) of the blood glucose levels were calculated. The patients were divided into low GV (SD $<2$ $\mathrm{mmol} / \mathrm{L})$ and high $\mathrm{GV}(\mathrm{SD} \geqslant 2 \mathrm{mmol} / \mathrm{L})$ groups according to a previously described method.

\section{Animal experiments}

\section{Animals}

The animal study was approved by the Xuanwu Hospital Institutional Animal Care and Use Committee and was conducted in accordance with the Guide for the Care and Use of Laboratory Animals (National Research Council, 8th edition, 2011 revision).

Five-month-old male miniature pigs weighing $32-40 \mathrm{~kg}$ $(\mathrm{n}=13)$ were purchased from China Agricultural University. The animal work was conducted by the Nongnong Biotechnology Company. The pigs were randomly assigned to sham control $(\mathrm{n}=3)$ and $\mathrm{DM}(\mathrm{n}=10)$ groups. The diabetic pigs were fed a high-sucrose and high-fat diet (37\% sucrose, $20 \%$ lard and $2 \%$ cholesterol) for 18 weeks, according to our previous study (9). The pigs in the sham control group were fed a normal diet. The establishment of a DM model was regarded as successful if the blood glucose consistently exceeded $150 \mathrm{mg} / \mathrm{dL}$. A schematic of the experimental procedures is shown in Figure 1.

\section{Stent implantation and grouping}

From the $19^{\text {th }}$ week, pigs with DM were administered clopidogrel $(300 \mathrm{mg})$ and aspirin $(300 \mathrm{mg})$ antiplatelet therapy. Twenty coronary sites for stent implantation in 10 diabetic pigs situated in the proximal to mid-section of the right coronary arteries (RCAs) and the left anterior descending (LAD) were selected after angiography. A total of 20 drug-eluting stents (XIENCE Xpedition, Abbott, USA) were implanted, with stent overstretching up to $20 \%$. Choutet $(10 \mathrm{mg} / \mathrm{kg}$ ) (Vick Ltd, France) was administered intramuscularly for anaesthesia, and isoflurane (3\%) (Ruiward Life Technology Co., Ltd, Shenzhen, China) was maintained using a ventilator. The procedure was supported by mechanical ventilation after anaesthetization.

Following stent implantation, interstitial glucose concentrations were determined from 19 to 40 weeks with the use of a glucose monitoring system (GMS) (Alameda, USA). This device measured glucose data automatically through subcutaneous filaments inserted beneath the internal femur skin of pigs. The sensor collected glucose measurements and trends every 15 minutes and stored up to $8 \mathrm{~h}$ of glucose readings. The mean blood glucose concentration and SD were automatically calculated. The diabetic pigs were subgrouped as low $\mathrm{GV}(\mathrm{SD}<3 \mathrm{mmol} / \mathrm{L})$ or high $\mathrm{GV}(\mathrm{SD} \geq 3 \mathrm{mmol} / \mathrm{L})$ accordingly.

\section{Optical coherence tomography (OCT)}

At 40 weeks, angiography and OCT were performed to examine the intima. The LightLab Imaging System (MA, USA) was used to determine the stent area (SA) and the lumen area (LA). The neointimal area (NIA) was calculated as NIA = LA - SA, and the percent area stenosis (\%AS) $=[1-(\mathrm{LA} / \mathrm{SA})] \times 100$. Neointimal thickness $(\mathrm{NIT})$ was determined by measuring the distance from the centre of each stent strut to the luminal border in the direction of the centre of gravity. The sham group did not undergo the stent implantation procedure.

\section{Biochemical, histological and molecular examination}

Blood samples were collected before and after the experiments. Fasting blood glucose (FBG) and the blood lipid profile, including total cholesterol (TC), high-density lipoprotein cholesterol (HDL-C), low-density lipoprotein cholesterol (LDL-C), and triglyceride (TG) levels were determined.

\section{Histological analysis and western blot}

After OCT examination, the pigs were euthanized by intravenous administration of $20 \mathrm{~mL}$ of $15 \%$ potassium chloride. Dissections were carried out to isolate porcine hearts 


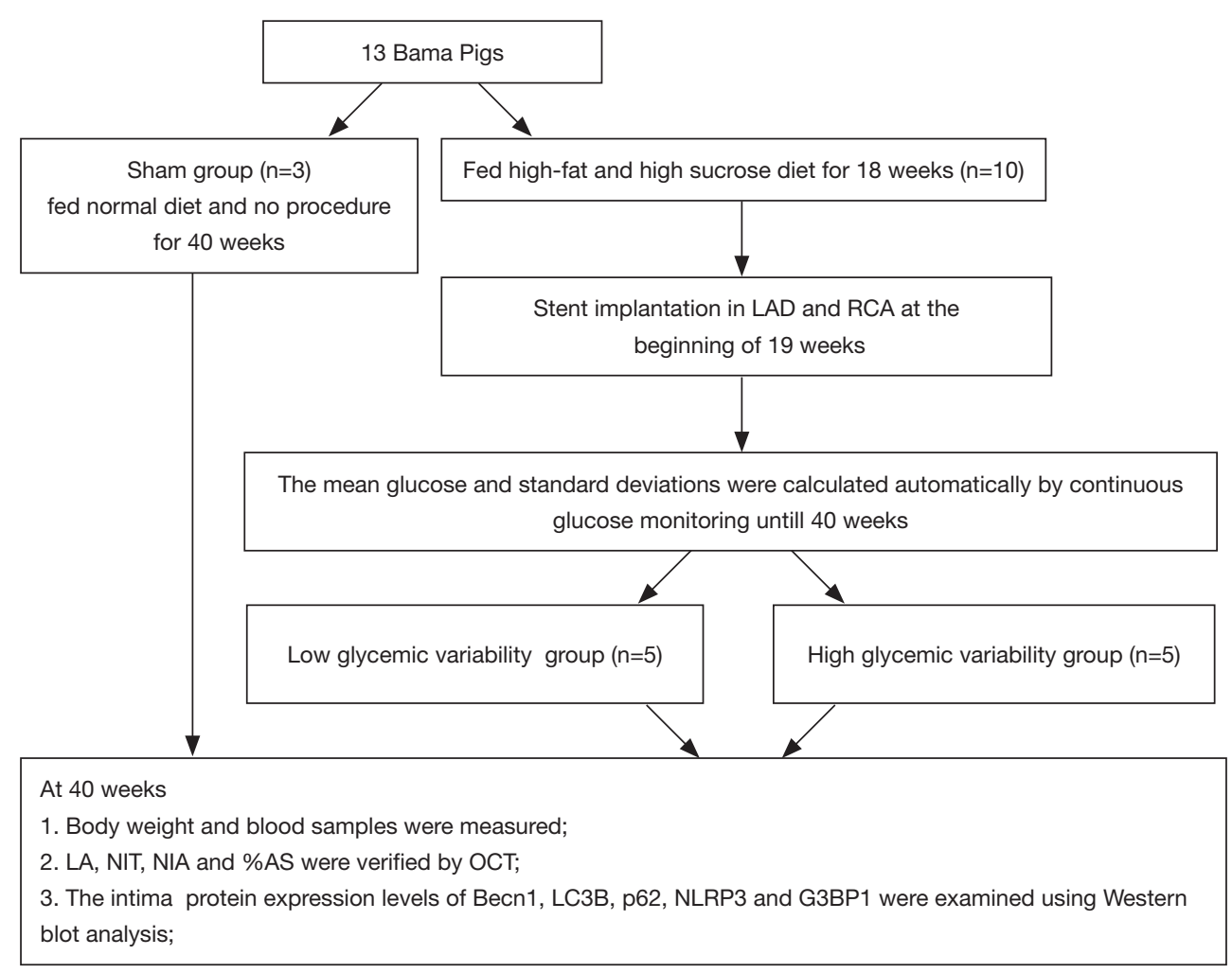

Figure 1 Study protocol. RCA, right coronary artery; LAD, left anterior descending; \%AS, percentage of area stenosis; NIT, neointimal thickness; LA, lumen area; NIA, neointimal area; OCT, optical coherence tomography.

and to separate the artery stent segments. After perfusion with $0.9 \%$ saline to wash out the blood, the coronaries were fixed with $10 \%$ formalin for at least $1 \mathrm{~h}$. For stented arterial segments, the tissues were fixed, dehydrated and embedded. Three cross sections, at the proximal, mid and distal segments (cutting thicknesses of 4 to $6 \mu \mathrm{m}$ ), were obtained from each specimen and were stained with haematoxylin and eosin (H\&E) and Movat's stain.

Total protein was extracted from the intima at the edge of the stent segment, in accordance with the previous study (10). The membrane was blocked with specific diluted $(1: 1,000)$ primary antibody at $4{ }^{\circ} \mathrm{C}$ overnight. Primary antibody against G3BP1 was purchased from Abcam (UK); antibodies against NLRP3, Becn1, LC3B and p62 were purchased from Novus (USA). $\beta$-actin (1:2,000; Anhui, China) served as a control. The membrane was washed and then incubated with corresponding secondary antibody for $1 \mathrm{~h}$.

\section{In vitro experiments}

The human monocytic leukaemia cell line THP-1 was obtained from the Cell Bank of the Type Culture Collection of the Chinese Academy of Sciences, Shanghai, China. Cells were cultured in RPMI 1640 medium (Gibco, USA) containing streptomycin $(100 \mu \mathrm{g} / \mathrm{mL}), \beta$-mercaptoethanol $(0.05 \mathrm{mM})$ (Sigma-Aldrich, USA) and foetal bovine serum (10\%) (Biological Industries, USA) at $37{ }^{\circ} \mathrm{C}$ with $5 \% \mathrm{CO}_{2}$. THP-1 monocytes $\left(1 \times 10^{6}\right.$ cells $\left./ \mathrm{mL}\right)$ were treated with phorbol-12-myristate acetate (PMA) (160 nM) (SigmaAldrich, USA) for 3 days to induce differentiation into macrophage-like cells.

The cells were cultured under different conditions. Cells that maintained in RPMI 1640 medium for $6 \mathrm{~h}$ were served as controls. Two groups of cells (low and high GV groups) were cultured in a low or high GV condition, as they were grown in routine RPMI 1640 medium and $5 \mathrm{~mol} / \mathrm{L} \mathrm{D}$-glucose (low GV) or $25 \mathrm{~mol} / \mathrm{L} \mathrm{D}$-glucose (high GV) alternately every 3 hours for 3 days. Another two groups of cells (high GV + rapamycin group and high $\mathrm{GV}+3$-methyladenine group) were pretreated with an autophagy inducer rapamycin (100 nmol/L; Selleck, USA) or an autophagy inhibitor 3 -methyladenine $(5 \mathrm{mmol} / \mathrm{L}$; 
Sigma-Aldrich, USA) for $24 \mathrm{~h}$, followed by cultured in the high $\mathrm{CV}$ condition mentioned above for 3 days. Western blot assay was utilized to quantified the expression levels of G3BP1 and NLRP3 in the above five groups.

\section{Statistical analysis}

The data were analysed with SPSS version 17.0. Continuous variables were reported as the mean $\pm \mathrm{SD}$ or the median $\left(25^{\text {th }}\right.$ and $75^{\text {th }}$ percentile) and were assessed for normality with the Shapiro-Wilk test. Normally distributed data were analysed by t-test and one-way ANOVA, while not normally distributed data were analysed by the Mann-Whitney and Kruskal-Wallis tests. Categorical variables are presented as numbers and percentages and were compared using the chisquared test or Fisher's exact test. A multivariate regression model was performed to identify predictors of RAMI. A two-tailed $\mathrm{P}$ value of $<0.05$ was considered statistically significant.

\section{Results}

\section{Clinical research}

In total, 95 consecutive patients were enrolled, with an average age of $62 \pm 11$ years. There was a slight predominance of men (67.4\%). The patients were divided as low GV ( $\mathrm{n}=61$ : type $2 \mathrm{DM} n=58$; type $1 \mathrm{DM} \mathrm{n}=3)$ and high $\mathrm{GV}(\mathrm{n}=34$ : type 2 $\mathrm{DM} n=32$; type $1 \mathrm{DM} \mathrm{n}=2$ ) groups. In the low $\mathrm{GV}$ group, 56 patients with type $2 \mathrm{DM}$ were treated with oral hypoglycaemic agents, and 2 patients with type $2 \mathrm{DM}$ were treated with oral hypoglycaemic agents and insulin subcutaneously. In the high GV group, 31 patients with type $2 \mathrm{DM}$ were treated with oral hypoglycaemic agents, and 1 patient with type 2 DM were treated with oral hypoglycaemic agents and insulin subcutaneously. During hospitalization, there were no differences between the two groups regarding age, gender, hypertension history, stroke, smoking, the proportion of type, treatment of DM, the Global Registry of Acute Coronary Events risk score, ejection fraction, left ventricular enddiastolic dimension, and the levels of HbA1c, blood lipids, serum creatinine, peak troponin I and $\mathrm{N}$-terminal pro-brain natriuretic peptide. After 2 years of follow-up, both groups presented equivalent levels of HbA1c, TC, LDL-C and HDL-C. The two groups showed similar mean blood glucose values. However, the $\mathrm{SD}$ values were higher in patients with high GV than in patients with low GV $(\mathrm{P}<0.01)$. There was a significantly increased incidence of RAMI in the high-GV group compared with the low-GV group (14.7\% vs. 1.6\%; $\mathrm{P}=0.038$ ). Based on the results of multivariate analysis, high GV was an independent predictor of RAMI (OR: 2.35, 95\% CI: $1.97-4.23, \mathrm{P}=0.03)$, representing a $55 \%$ incremental risk during 2 years of follow-up. The baseline characteristics during hospitalization and at the 2-year follow up are displayed in Table 1.

\section{Animal experiments}

\section{Body weight and biochemical parameters}

All the animal models survived until the end of the 40-week experimental period. The blood glucose concentrations were between 7.0 and $16.3 \mathrm{mmol} / \mathrm{L}$; thus, no insulin or oral hypoglycaemic drugs were administered. The body weight, FBG, TC, LDL-C and TG values were significantly higher in the $\mathrm{DM}$ group than in the sham group $(\mathrm{P}<0.05)$. No significant difference in HDL-C was observed between the diabetic and sham groups $(\mathrm{P}>0.05)$. The body weight, FBG and blood lipid profile were similar in the high- and lowGV groups $(\mathrm{P}>0.05)$. The GMS data showed that the DM group with high GV showed a similar mean blood glucose value but a significantly higher SD compared with the DM group with low GV (Table 2).

\section{OCT analysis}

As shown in Table 2, at 40 weeks there were significant differences between the high-GV and low-GV groups in some measures of OCT, such as LA, NIT, NIA and \%AS $(\mathrm{P}<0.05)$, with the exception of SA. The images of vessels by OCT and the histological findings by H\&E and Movat's staining are presented in Figure 2.

\section{Western blot analysis of NLRP3, G3BP1, Becn1, LC3B and $\mathrm{p} 62$}

NLRP3, G3BP1, Becn1, LC3B and p62 were more highly expressed in the DM group than in the sham group $(\mathrm{P}<0.05)$. Compared to the low-GV group, the high-GV group exhibited significantly higher protein expression levels of NLRP3 and p62 $(\mathrm{P}<0.05)$ but lower expression levels of Becn1, LC3B and G3BP1 $(\mathrm{P}<0.05)$. Figure 3 shows representative western blot images.

\section{In vitro experiments}

Compared to the control, GV, whether low or high, contributed to the increased expression of NLRP3 and G3BP1 $(\mathrm{P}<0.05)$. The high-GV group exhibited lower 
Table 1 Baseline characteristics/clinical factors before discharge and comparison of lipid, HbA1c, SD and RAMI during 2-year follow-up

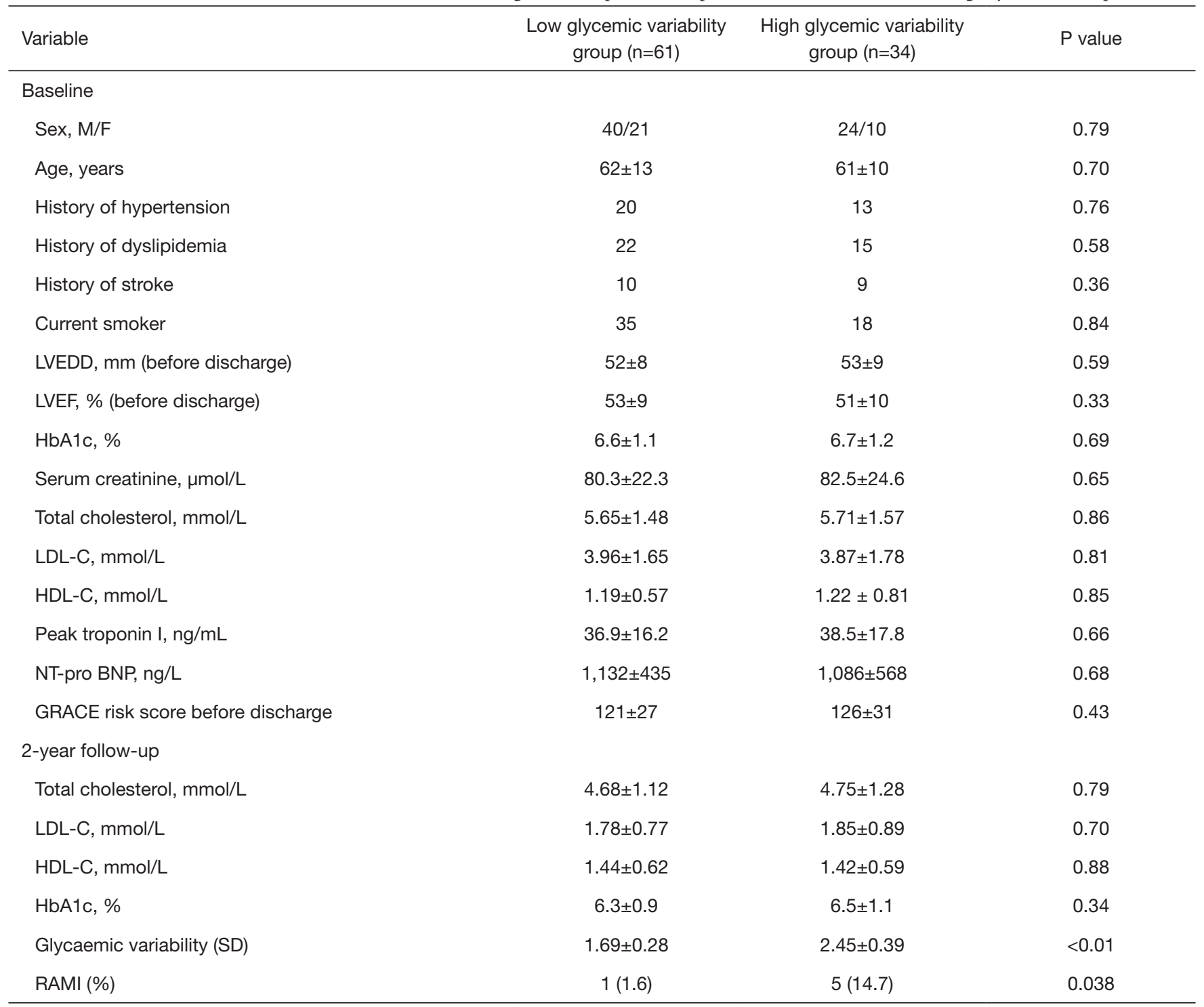

Values are given as $\mathrm{n}(\%)$ or mean \pm SD. M, male; F, female; LVEDD, left ventricular end diastolic diameter; LVEF, left ventricular ejection fraction; HbA1c, glycated hemoglobin; LDL-C, low-density lipoprotein cholesterol; HDL-C, high-density lipoprotein cholesterol; NT-pro BNP, N-terminal pro-brain natriuretic peptide; GRACE, Global Registry of Acute Coronary Events; SD, standard deviation; RAMI, recurrent acute myocardial infarction.

G3BP1 protein expression but higher NLRP3 expression compared to the low-GV group $(\mathrm{P}<0.05)$. The effects of high GV could be abolished by rapamycin, while they were aggravated by 3 -methyladenine. Representative western blot images are displayed in Figure 4.

\section{Discussion}

This study explored the effects of GV on intimal hyperplasia and plaque stability after coronary stenting and the mechanism of potential interplay signalling between autophagy and the G3BP1/NLRP3 inflammasome. The findings demonstrated that (I) high GV contributed to an increased risk of RAMI compared with low GV; (II) high GV was associated with higher degrees of NIT, NIA and $\% A S$ and lower LA compared with low GV; (III) high GV was associated with decreased Becn1, LC3B, and G3BP1 expression and increased p62 and NLRP3 expression; and 
Table 2 Comparison of body weight, blood samples and intimal hyperplasia assessed by OCT

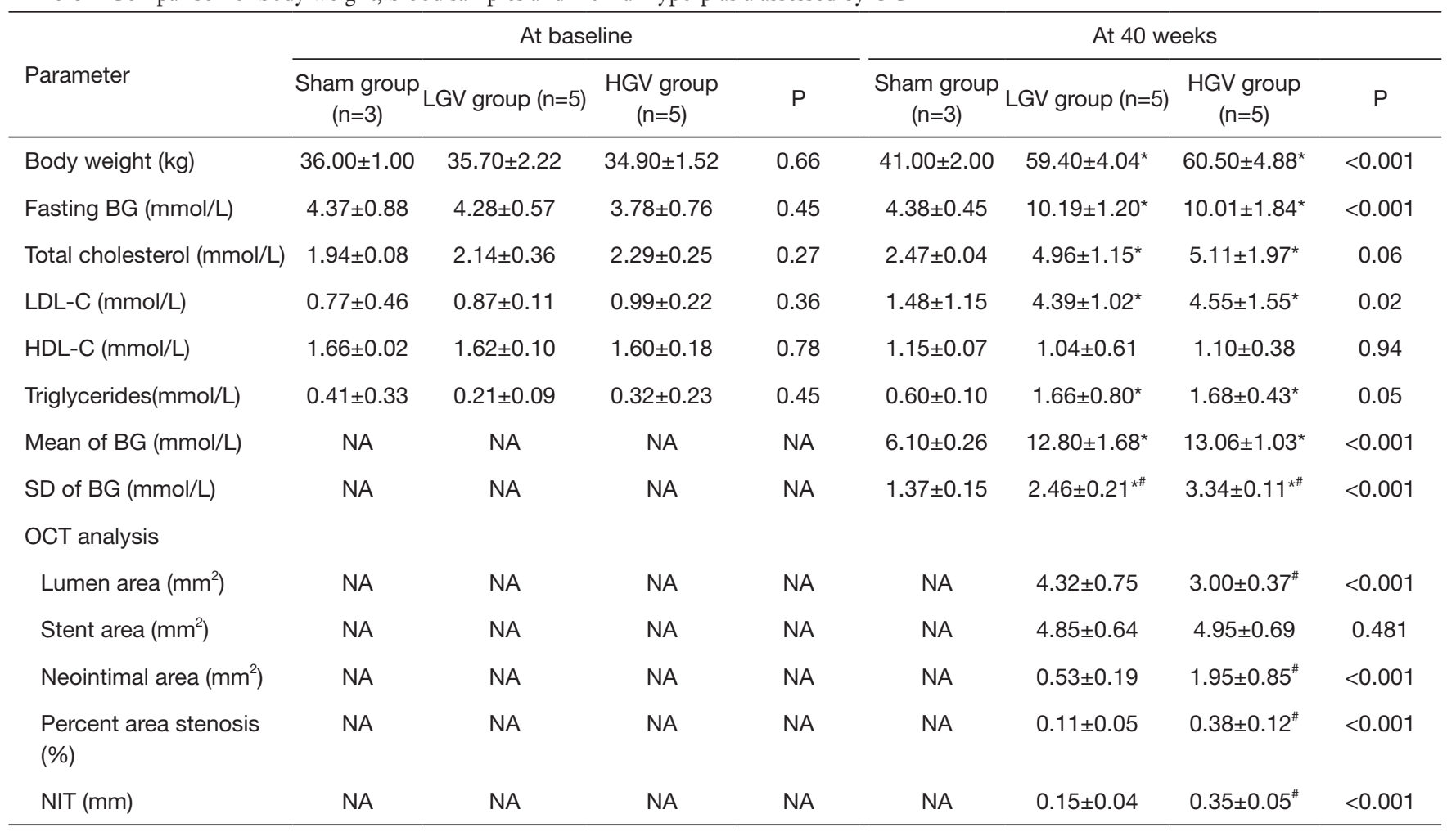

Data are presented as the mean \pm standard deviation. ${ }^{*} \mathrm{P}<0.05$, compared with sham group; ${ }^{*} \mathrm{P}<0.05$, compared with LGV group. OCT, optical coherence tomography; LGV, low glycemic variability; HGV, high glycemic variability group; BG, blood glucose; LDL-C, low-density lipoprotein cholesterol; HDL-C, high-density lipoprotein cholesterol; SD, standard deviations; NIT, neointimal thickness.

(IV) the effects of GV on G3BP1/NLRP3 inflammasome signalling in macrophages were dependent on the regulation of autophagy. This is the first study demonstrating the effects of $\mathrm{GV}$ on the progression of coronary atherosclerosis in clinical patients, large animals and cell experiments. This study also explored the mechanism by which this effect occurs via the relationship between autophagy and G3BP1/ NLRP3 inflammasome signalling.

We studied the impact of GV on intimal hyperplasia and plaque stability after stent implantation in clinical and animal studies. Our findings indicate that GV may be related to the occurrence, development and rupture of atherosclerotic plaques; this idea is supported by previous studies. A recent meta-analysis (11) showed that high GV may be more closely related to unfavourable outcomes in patients with CHD irrespective of CHD subtype and the presence or absence of diabetes. GV is associated with coronary plaque vulnerability at the culprit lesions and also at the non-culprit vessels $(12,13)$. The Kuroda study (14) showed that $\mathrm{GV}$ is associated with maximum neointimal thickness and with variability in neointimal thickness, although HbA1c levels did not differ significantly between groups. As shown in the current study, HbAlc values of the patients were similar at baseline and at the 2-year followup. Furthermore, the average blood glucose values were not significantly different in the experimental animals. However, the daily glycaemic profiles regarding the frequency, amplitude and duration of glycaemic excursions differed considerably in patients with diabetes with the same HbA1c level. The results also suggest that GV may be more harmful than chronic sustained hyperglycaemia. Glycaemic variability, as a new index of diabetes control, can supplement the deficiencies of HbA1c monitoring.

We further studied the possible mechanisms by which GV affects atherosclerosis by conducting large animal model and cell culture experiments. We found that higher GV was associated with increased NLRP3 expression. The aetiology of CHD, particularly AMI, is thought to be related to lipid metabolic disorders and chronic inflammation. Atherosclerosis, as a progressive chronic 

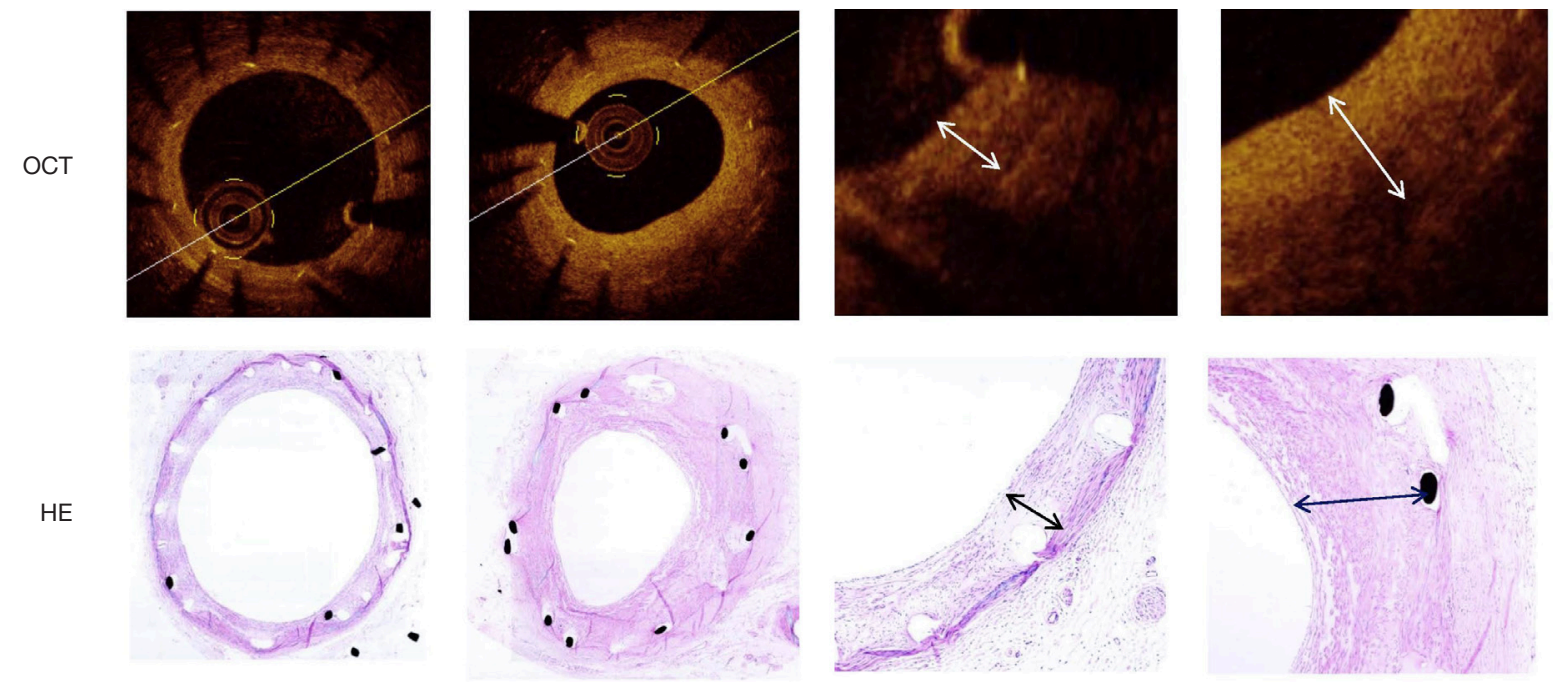

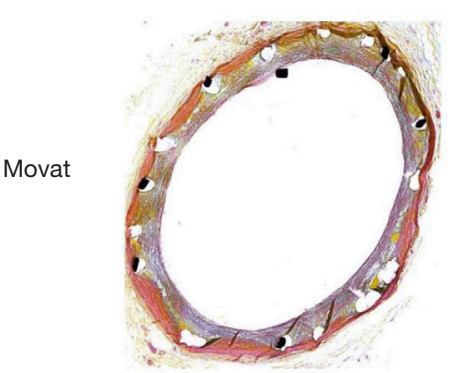

LGV group

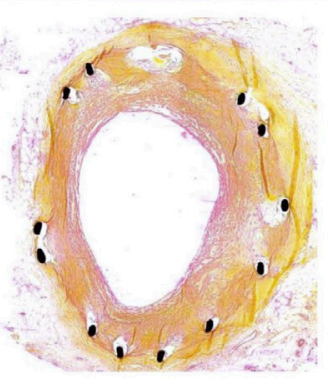

HGV group

Scale bar $200 \mu \mathrm{m}$

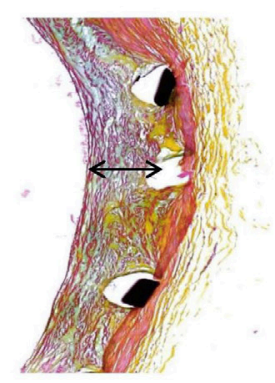

LGV group

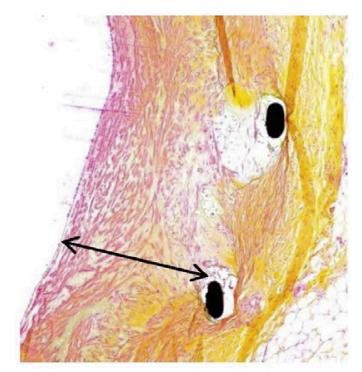

HGV group Scale bar $100 \mu \mathrm{m}$

Figure 2 OCT images and histological findings of vessels stained with H\&E and Movat staining in the LGV and HGV groups. OCT, optical coherence tomography; LGV, low glycaemic variability; HGV, high glycaemic variability.

inflammation disorder, is characterized by endothelial dysfunction, the aberrant proliferation of vascular smooth muscle cells, leukocyte infiltration, and the accumulation of lipids in arteries (15). A previous study by Duewell et al. (16) demonstrated the involvement of the NLRP3 inflammasome in diet-induced atherosclerosis in mice via the activation of caspase-1 by upregulated NLRP3 inflammasome activity in macrophages. We found that higher GV was associated with lower G3BP1 expression levels. Stress granules promote cell survival in response to a wide range of stimuli by interfering with the NLRP3 inflammasome (5). The study by Herman (17) identified the formation of stress granules that accumulated in intimal macrophages during atherosclerosis progression. Our results are supported by this recent study. Our findings also suggest that high $\mathrm{GV}$ might abolish the homeostasis of the inflammatory microenvironment, enhancing a proinflammatory effect and weakening an anti-inflammatory effect. We further investigated how autophagy regulates stress granules/NLRP3 inflammasome signalling. We found that rapamycin abolished the impact of high-glycaemic variability, while 3 -methyladenine aggravated the impact of high-glycaemic variability. Autophagy is a complicated intracellular process in which injured cytoplasmic constituents or superfluous organelles are digested and removed within lysosomes through the action of hydrolytic enzymes (18). The accumulation of macrophages is involved in the formation, growth and instability of atherosclerotic plaques (19). Under stimulation by oxidative stress or LDL-C, autophagy becomes activated in macrophages, where it degrades and removes dysfunctional organelles and misfolded proteins, enhances macrophage efferocytosis, and strengthens cholesterol transport from macrophage-derived foam cells in various stages of atherosclerosis (20). The 


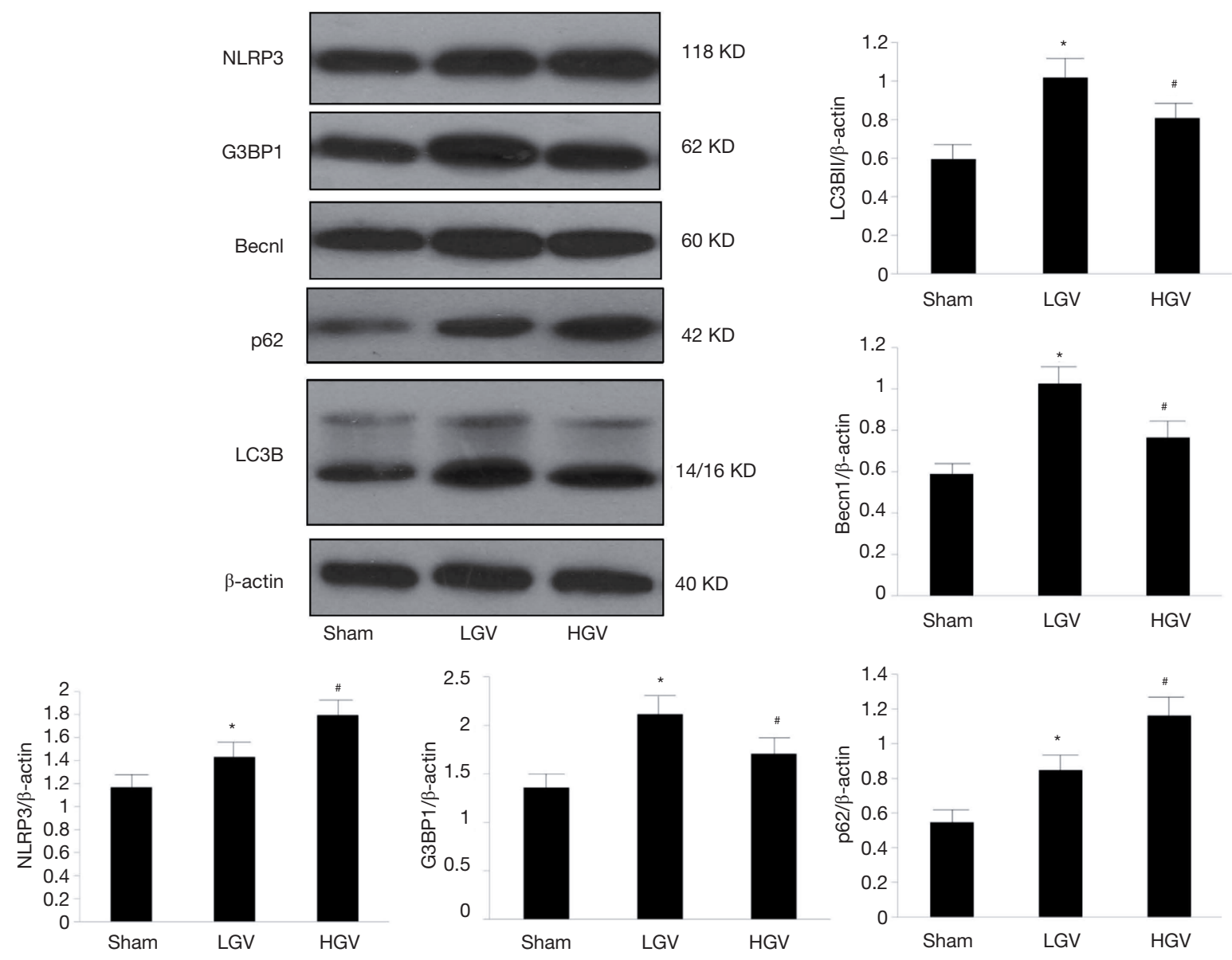

Figure 3 Western blot analysis of G3BP1, NLRP3, Becn1, LC3B and p62 in the Sham, LGV and HGV groups. LGV, low glycaemic variability; HGV, high glycaemic variability. Comparisons were made in three groups $(\mathrm{n}=3$ in each). The results were reported as the mean \pm SD. ${ }^{*} \mathrm{P}<0.05$ vs. LGV group, ${ }^{*} \mathrm{P}<0.05$ vs. sham group. Densities of bands were normalized to the $\beta$-actin.

study by Munasinghe showed that autophagy was markedly increased in diabetic heart, accompanied by the increased expression of LC3B-II, an autophagy marker, along with its mediator Beclin-1 (21). The NLRP3 inflammasome can be degraded through an autophagic process during the development of atherosclerosis (22-24). Our results suggest that higher GV is associated with reduced autophagy, which in turn increases inflammation. Autophagy can engulf NLRP3 through recognizing p62 of the K63 ubiquitin chains on NLRP3, suggesting that manipulating autophagy would be a potential approach to inhibit NLRP3-related inflammation (22). The relationship between autophagy and stress granules is a new finding in our study. However, it remains unclear whether autophagy directly regulates stress granules or indirectly regulates stress granules by regulating the NLRP3 inflammasome.
This study has some limitations. The clinical research portion contained a small sample size and did not measure mechanism-related markers, such as LC3B, Beclin-1, NLRP3 and G3BP1. In the large animal experiments, we did not establish a hyperlipidaemia control group. In vitro, an analysis of more inflammatory markers may have helped to better clarify the involved mechanisms.

\section{Conclusions}

Glycaemic variability might impact intimal hyperplasia and plaque stability via autophagy-mediated G3BP1/ NLRP3 inflammasome signalling. Therefore, GV and autophagy-mediated G3BP1/NLRP3 inflammasome signalling might be novel promising targets for CHD treatment. 


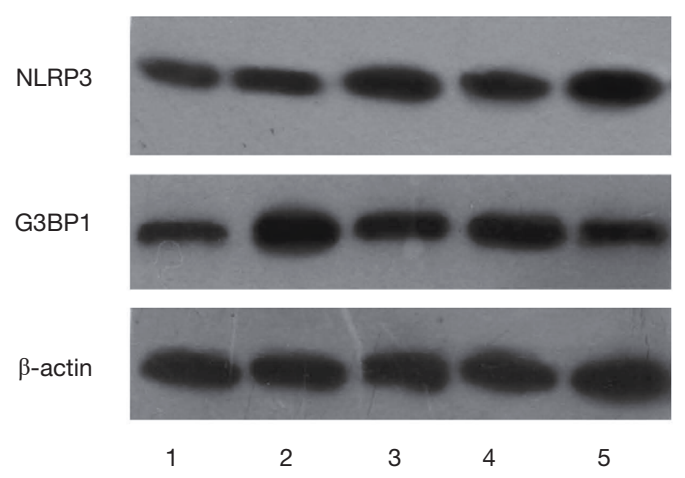

$118 \mathrm{KD}$

$62 \mathrm{KD}$

$40 \mathrm{KD}$
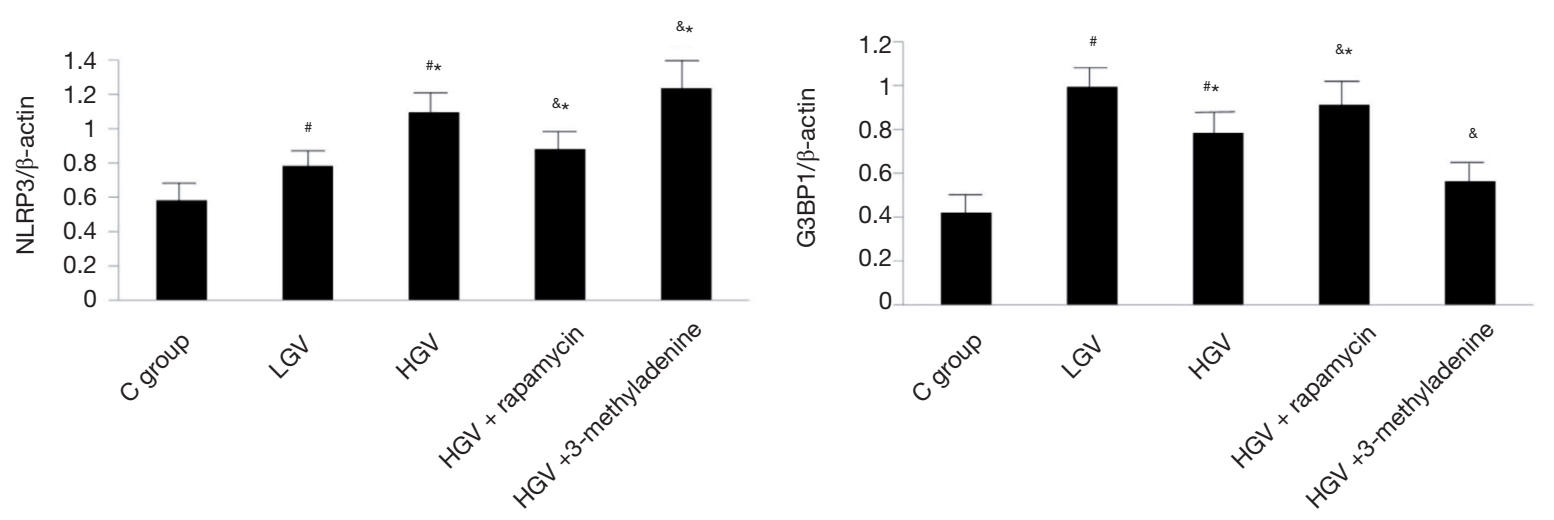

Figure 4 Western blot analysis of NLRP3 and G3BP1 in the control group, low glycaemic variability group, high glycaemic variability group, high glycaemic variability + rapamycin group and high glycaemic variability +3 -methyladenine group. Comparisons were made in five groups ( $\mathrm{n}=3$ in each). ${ }^{*} \mathrm{P}<0.05$ vs. sham group, ${ }^{\#} \mathrm{P}<0.05$ vs. LGV group, ${ }^{\circledR} \mathrm{P}<0.05$ vs. HGV group. The results were presented as the mean \pm SD. Densities of bands were normalized to the $\beta$-actin.

\section{Acknowledgments}

Funding: This work was supported by the National Natural Science Foundation of China (Grant number 81770344), the National Clinical Research Center for Geriatric Diseases, the Beijing Key Clinical Speciality Development Project and the China Young and Middle-aged Clinical Research VG fund (Grant number 2017-CCA-VG-043).

\section{Footnote}

Reporting Checklist: The authors have completed the ARRIVE reporting checklist. Available at http://dx.doi. org/10.21037/atm-20-4818

Data Sharing Statement: Available at http://dx.doi. org/10.21037/atm-20-4818
Conflicts of Interest: All authors have completed the ICMJE uniform disclosure form (available at http://dx.doi. org/10.21037/atm-20-4818). The authors have no conflicts of interest to declare.

Ethical Statement: The authors are accountable for all aspects of the work in ensuring that questions related to the accuracy or integrity of any part of the work are appropriately investigated and resolved. All procedures were conducted in compliance with the Declaration of Helsinki (as revised in 2013). The Xuanwu Hospital Ethics Committee approved the study protocols (ID: 2017-042) and written informed consent was provided by patients before participating in the study. The animal study was approved by the Xuanwu Hospital Institutional Animal Care and Use Committee, and was conducted in accordance with the Guide for the Care and Use of Laboratory Animals 
(National Research Council, 8th edition, 2011 revision).

Open Access Statement: This is an Open Access article distributed in accordance with the Creative Commons Attribution-NonCommercial-NoDerivs 4.0 International License (CC BY-NC-ND 4.0), which permits the noncommercial replication and distribution of the article with the strict proviso that no changes or edits are made and the original work is properly cited (including links to both the formal publication through the relevant DOI and the license). See: https://creativecommons.org/licenses/by-nc-nd/4.0/.

\section{References}

1. Xia J, Xu J, Hu S, et al. Impact of glycaemic variability on the occurrence of periprocedural myocardial infarction and major adverse cardiovascular events (MACE) after coronary intervention in patients with stable angina pectoris at 6months follow-up. Clin Chim Acta 2017;471:196-200.

2. Klionsky DJ, Emr SD. Autophagy as a regulated pathway of cellular degradation. Science 2000;290:1717-21.

3. Yan L, Vatner DE, Kim SJ, et al. Autophagy in chronically ischemic myocardium. Proc Natl Acad Sci U S A 2005;102:13807-12.

4. Khan S, Salloum F, Das A, et al. Rapamycin confers preconditioning-like protection against ischemiareperfusion injury in isolated mouse heart and cardiomyocytes. J Mol Cell Cardiol 2006;41:256-64.

5. Samir P, Kesavardhana S, Patmore DM, et al. DDX3X acts as a live-or-die checkpoint in stressed cells by regulating NLRP3 inflammasome. Nature 2019;573:590-4.

6. Nielsen CT, Østergaard O, Rasmussen NS, et al. A review of studies of the proteomes of circulating microparticles: key roles for galectin-3-binding protein-expressing microparticles in vascular diseases and systemic lupus erythematosus. Clin Proteomics 2017;14:11.

7. Satish M, Agrawal DK. Atherothrombosis and the NLRP3 inflammasome - endogenous mechanisms of inhibition. Transl Res 2020;215:75-85.

8. Jiang C, Jiang L, Li Q, et al. Acrolein induces NLRP3 inflammasome-mediated pyroptosis and suppresses migration via ROS-dependent autophagy in vascular endothelial cells. Toxicology 2018;410:26-40.

9. Xia J, Qu Y, Yin C, et al. Optical Coherence Tomography Assessment of Glucose Fluctuation Impact on the Neointimal Proliferation After Stent Implantation in a Diabetic/Hypercholesterolemic Swine Model. Int Heart J
2017;58:608-14.

10. Xia JG, Xu FF, Qu Y, et al. Atorvastatin post-conditioning attenuates myocardial ischemia reperfusion injury via inhibiting endoplasmic reticulum stress-related apoptosis. Shock 2014;42:365-71.

11. Pu Z, Lai L, Yang $X$, et al. Acute glycaemic variability on admission predicts the prognosis in hospitalized patients with coronary artery disease: a meta-analysis. Endocrine 2020;67:526-34.

12. Teraguchi I, Imanishi T, Ozaki Y, et al. Impact of glucose fluctuation and monocyte subsets on coronary plaque rupture. Nutr Metab Cardiovasc Dis 2014;24:309-14.

13. Gohbara M, Hibi K, Mitsuhashi T, et al. Glycaemic Variability on Continuous Glucose Monitoring System Correlates With Non-Culprit Vessel Coronary Plaque Vulnerability in Patients With First-Episode Acute Coronary Syndrome - Optical Coherence Tomography Study. Circ J 2016;80:202-10.

14. Kuroda M, Shinke T, Otake H, et al. Effects of daily glucose fluctuations on the healing response to everolimuseluting stent implantation as assessed using continuous glucose monitoring and optical coherence tomography. Cardiovasc Diabetol 2016;15:79.

15. Kang J, Sun Y, Deng Y, et al. Autophagy-endoplasmic reticulum stress inhibition mechanism of superoxide dismutase in the formation of calcium oxalate kidney stones. Biomed Pharmacother 2020;121:109649.

16. Duewell P, Kono H, Rayner KJ, et al. NLRP3 inflammasomes are required for atherogenesis and activated by cholesterol crystals. Nature 2010;464:1357-61.

17. Herman AB, Silva Afonso M, Kelemen SE, et al. Regulation of Stress Granule Formation by Inflammation, Vascular Injury, and Atherosclerosis. Arterioscler Thromb Vasc Biol 2019;39:2014-27.

18. Hassanpour M, Rahbarghazi R, Nouri M, et al. Role of autophagy in atherosclerosis: foe or friend. J Inflamm (Lond) 2019;16:8.

19. Xu H, Jiang J, Chen W, et al. Vascular Macrophages in Atherosclerosis. J Immunol Res 2019;2019:4354786.

20. Shao BZ, Han BZ, Zeng YX, et al. The roles of macrophage autophagy in atherosclerosis. Acta Pharmacol Sin 2016;37:150-6.

21. Munasinghe PE, Riu F, Dixit P, et al. Type-2 diabetes increases autophagy in the human heart through promotion of Beclin-1 mediated pathway. Int J Cardiol 2016;202:13-20.

22. Zhou Z, Zhu X, Yin R, et al. K63 ubiquitin chains target NLRP3 inflammasome for autophagic degradation in ox- 
Page 12 of 12

LDL-stimulated THP-1 macrophages. Aging (Albany NY) 2020;12:1747-59.

23. Wang $\mathrm{Y}$, Meng $\mathrm{C}$, Zhang J, et al. Inhibition of GSK-3 $\beta$ alleviates cerebral ischemia/reperfusion injury in rats by suppressing NLRP3 inflammasome activation through

Cite this article as: Xia J, Zhang J, Chang J, Tian Y, Li J, Zhang B, Zeng X, Yin C. The effects of glycaemic variability on intimal hyperplasia and plaque stability after stenting via autophagy-mediated G3BP1/NLRP3 inflammasome. Ann Transl Med 2020;8(21):1388. doi: 10.21037/atm-20-4818
Xia et al. The effects of glycaemic variability on atherosclerosis

autophagy. Int Immunopharmacol 2019;68:234-41.

24. Ma S, Chen J, Feng J, et al. Melatonin Ameliorates the Progression of Atherosclerosis via Mitophagy Activation and NLRP3 Inflammasome Inhibition. Oxid Med Cell Longev 2018;2018:9286458. 